\title{
Channel State Feedback over the MIMO-MAC
}

\author{
K. Raj Kumar and Giuseppe Caire, Fellow, IEEE
}

\begin{abstract}
We consider the problem of designing low latency and low complexity schemes for channel state feedback over the MIMO-MAC (multiple-input multiple-output multiple access channel). We develop a framework for analyzing this problem in terms of minimizing the MSE distortion, and come up with separated source-channel schemes and joint source-channel schemes that perform better than analog feedback. We also develop a strikingly simple code design based on scalar quantization and uncoded QAM modulation that achieves the theoretical asymptotic performance limit of the separated approach with very low complexity and latency, in the case of single-antenna users.
\end{abstract}

\section{Index Terms}

Multiple-Antenna Multiple-Access Channel, Channel State Feedback, Wireless Communications

\section{INTRODUCTION}

$\mathbf{C}$ ONSIDER a frequency division duplex (FDD) cellular system with sufficient frequency spacing between the uplink and downlink channels, such that the uplink and downlink fading coefficients are independent. A base station (BS) with $M$ antennas wishes to serve $K$ user terminals (UTs), with $N_{t}$ antennas each, using some MIMO broadcast channel precoding technique, such as linear beamforming, Dirty-Paper Coding or some low-complexity non-linear precoding approximation thereof. Essential to these techniques is the availability of accurate channel state information at the transmitter (CSIT), that is, the BS must know the user downlink channels. We consider the popular block-fading model, according to which the channel coefficients remain constant for time-frequency slots of some finite but large number of channel uses (complex dimensions), and change independently from slot to slot. We assume that the UTs have perfect knowledge of their downlink channels on each block, which can be obtained from downlink training symbols broadcasted by the BS. Then, at each time slot, the downlink channel coefficients must be fed back to the BS on the uplink. We can model this CSIT feedback as signaling over a MIMO multiple-access channel (MAC). It has been shown in [8], [9] that the relevant performance measure that dominates the downlink rate gap ${ }^{1}$ is the mean-square error (MSE) distortion at which the BS is able to represent the UTs channel coefficients (this will be made more precise in the sequel). It follows that the CSIT feedback problem consists of lossy transmission under an end-to-end MSE distortion constraint of

K. R. Kumar is with the Laboratoire d'algorithmique, Ecole Polytechnique Fédérale de Lausanne, 1015 Lausanne, Switzerland (raj.kumardepfl.ch). G. Caire is with the Department of Electrical Engineering - Systems, University of Southern California, Los Angeles, CA 90089, USA (caire@usc.edu). Much of this work was carried out while K. R. Kumar was with the University of Southern California.

The material in this paper was presented in part at the IEEE International Symposium on Information Theory (ISIT-09), Seoul, Korea, Jun. 28 - Jul. 3, 2009.

${ }^{1}$ The gap between the rate achievable with imperfect CSIT from the optimal rate achievable with perfect CSIT and the optimal MIMO broadcast channel coding strategy. 
a set of $K$ Gaussian sources (i.e., the UTs downlink channel coefficients) over a MIMO-MAC channel affected by block fading. It should also be noticed that, apart from some "analog" feedback schemes where the channel coefficients are sent unquantized and in parallel by all users (see [8], [9] and references therein), very little attention has been devoted to properly design the CSIT feedback scheme exploiting the MIMO-MAC nature of the uplink channel: most works assume perfect feedback at fixed rate, or implicitly assume that the feedback information is piggybacked "somehow" into the uplink transmissions. This may pose problems, since the CSIT feedback must have extremely low latency, therefore, its coding block length must be very short. In this paper we consider the problem of designing very low latency and low complexity CSIT feedback schemes for the uplink MIMO-MAC.

\section{Channel Model and Problem Statement}

Although the fading slots may span a large number of channel uses, we focus here only on the dimensions effectively used by the CSIT feedback scheme. We assume that the CSIT feedback uplink transmission spans a block of $T$ channel uses of the complex baseband equivalent MIMO-MAC channel, represented by

$$
\mathbf{Y}=\sqrt{\rho} \sum_{i=1}^{K} \mathbf{H}_{i} \mathbf{X}_{i}+\mathbf{W}
$$

where $\mathbf{Y}$ denotes the $M \times T$ signal received at the $\mathrm{BS}, \mathbf{X}_{i}$ is the $N_{t} \times T$ signal transmitted by the $i^{\text {th }}$ $\mathrm{UT}, \mathbf{H}_{i}$ is the (uplink) channel between the $i^{\text {th }} \mathrm{UT}$ and the BS that is assumed to remain constant over $T$ channel uses, and $\mathbf{W}$ is the additive noise. We assume that the entries of $\mathbf{H}_{i}$ and $\mathbf{W}$ are distributed as i.i.d. complex Gaussian with zero mean and unit variance $\mathcal{C N}(0,1)$. As anticipated before, we assume that the channels remain constant for a such block of $T$ channel uses. We impose an average per-user power constraint

$$
\mathbb{E}\left[\left\|\mathbf{X}_{i}\right\|_{F}^{2}\right] \leq T \quad \forall i
$$

where $\|\cdot\|_{F}$ denotes the Frobenius norm. Hence $\rho$ takes on the meaning of the uplink transmit SNR.

We consider that each UT needs to transmit $S$ samples of a complex i.i.d. Gaussian source ${ }^{2}$ over $T$ uses of the MIMO-MAC. We define $b \triangleq T / S$ as the bandwidth efficiency of the feedback scheme, measured as the number $b$ of feedback (uplink) channel uses per source sample (i.e., per downlink channel coefficient). Therefore, it is clear that the bandwidth efficiency immediately relates to the "protocol overhead" incurred by a closed-loop channel state feedback scheme. Associated with a particular $b$, we consider a family of coding schemes $\mathcal{C}(\rho)$ indexed by the SNR $\rho$. Corresponding to the coding scheme $\mathcal{C}(\rho)$, we define $D(\rho)$ to be the MSE distortion involved in reproducing the source, averaged over the source, channel and the noise. The distortion SNR exponent of the family is given by [4]-[6]

$$
\delta(b)=-\lim _{\rho \rightarrow \infty} \frac{\log D(\rho)}{\log \rho} .
$$

The distortion SNR exponent of the channel $\delta^{*}(b)$ is given by the supremum of $\delta(b)$ over all possible coding schemes.

The operational significance of the distortion exponent defined above for the CSIT feedback problem is provided next, as the main motivation for the results of this paper. The key quantity that dominates the

\footnotetext{
${ }^{2}$ The source length $S$ corresponds to the overall number of channel coefficients that need to be fed back at any time slot.
} 
rate gap in the MIMO downlink with imperfect CSIT is the system self-interference, due to imperfect cancellation of the multiuser interference due to the imperfect knowledge of the downlink channels. The self-interference average power is given by the product between the downlink transmitted signal power and the channel MSE distortion at the BS. Up to constants, this is given by [8], [9] $\rho \cdot D(\rho)$, provided that the uplink and the downlink SNRs differ by a constant factor. In the high-SNR limit $(\rho \rightarrow \infty)$, if the above product vanishes, then the asymptotic high-SNR rate gap goes to zero and the imperfect CSIT scheme yields close-to-optimal performance. If the above product converges to a constant, then a constant rate gap is achieved. If the above product grows as a power $\rho^{\mu}$, with $0 \leq \mu \leq 1$, then the downlink sum-rate scales only as $\min \left\{M, K N_{t}\right\}(1-\mu) \log \rho$, i.e., a loss of degrees of freedom by the factor $1-\mu$ occurs. Finally, if the above product grows as $\rho$ (i.e., $D(\rho)=O(1)$ ), then the user rates saturate to a constant term and the system becomes self-interference limited (unless only a single user is served at each slot, of course). It is therefore clear that the MIMO downlink performance depends critically on the distortion exponent $\delta(b)$.

From a practical viewpoint it should be noticed that the dynamic range of SNR in a cellular system in the presence of distance-dependent pathloss may be of the order of $40 \mathrm{~dB}$, i.e., the receiver SNR of UTs near the BS may be $40 \mathrm{~dB}$ larger than the received SNR of UTs at the cell edge. Hence, it is essential to design a CSIT feedback scheme whose MSE distortion $D(\rho)$ improves with the received SNR $\rho$. Schemes based on quantization codebooks of fixed size, provide $D(\rho)=O(1)$ (i.e., $\delta(b)=0$ for any $b$ ). The analog feedback scheme (see [8], [9] and references therein) achieves $\delta(b)=1$ for all $b \geq 1$. This is optimal for $b=1$, but generally suboptimal for $b>1$. Our goal is to study more efficient techniques that achieve $\delta(b)>1$ at some $b \geq 1$.

We first present an upper bound on the distortion SNR exponent in Sec. III and then analyze the performance of a separated source-channel coding scheme in Sec. IV. The sub-optimality of the separated scheme prompts us to investigate joint source-channel coding schemes for the MIMO-MAC, following which we present a hybrid digital-analog coding scheme in Sec. V. In Sec. VI, we construct simple channel codes that have optimal performance for the important special case where the UTs have a single antenna each. Simulation results that highlight the superiority of digital feedback over conventional analog feedback, and establish the relevance of the distortion SNR exponent in practical scenarios are presented in Sec. VII.

\section{UPPER BOUND ON THE DISTORTION SNR EXPONENT}

Our upper bound is inspired by the one presented for the single-user MIMO block fading channel in [4], [6]. First, notice that we consider the simple case of UTs with symmetric statistics, and since the UTs have no uplink channel state information, they all send the same coding rate, by symmetry. Define $\mathbf{H}=\left[\mathbf{H}_{1} \mathbf{H}_{2} \cdots \mathbf{H}_{K}\right]$. Consider an augmented channel where all UTs are provided with the knowledge of the coding rate $R(\mathbf{H})$ that can be transmitted such that the rate point $\mathbf{R}(\mathbf{H})=(R(\mathbf{H}), \ldots, R(\mathbf{H}))$ is inside the capacity region of the MIMO-MAC with given channel vectors and transmit SNR $\rho$, denoted by $\mathcal{C}_{\text {MAC }}(\mathbf{H} ; \rho)$. Using this knowledge, each UT can employ a separated source-channel coding scheme with a source coding rate $R_{s}=b R(\mathbf{H})$ nats per complex sample, and achieve an end-to-end instantaneous distortion given by $\mathcal{D}(\mathbf{H})=\exp (-b R(\mathbf{H}))$. This would result in the average distortion $D(\rho)=\mathbb{E}[\exp (-b R(\mathbf{H}))]$. 
From the expression of the MIMO-MAC capacity region $\mathcal{C}(\mathbf{H} ; \rho)$ we have the following conditions on the rate $R(\mathbf{H})$,

$$
R(\mathbf{H}) \leq \frac{1}{|\mathcal{K}|} \log \operatorname{det}\left(\mathbf{I}+\rho \sum_{k \in \mathcal{K}} \mathbf{H}_{k} \mathbf{H}_{k}^{\mathrm{H}}\right)
$$

for all subsets $\mathcal{K} \subseteq\{1, \ldots, K\}$, where $|\mathcal{K}|$ denotes the cardinality of the set $\mathcal{K}$. Denote $\mathbf{H}_{\mathcal{K}}$ to be the equivalent $M \times|\mathcal{K}| N_{t}$ channel comprising of the channels of the users in $\mathcal{K}$ stacked next to each other. We can rewrite (2) as

$$
R(\mathbf{H}) \leq \frac{1}{|\mathcal{K}|} \log \operatorname{det}\left(\mathbf{I}+\rho \mathbf{H}_{\mathcal{K}} \mathbf{H}_{\mathcal{K}}^{\mathrm{H}}\right)
$$

Let $m_{\mathcal{K}} \triangleq \min \left\{M,|\mathcal{K}| N_{t}\right\}$ and $n_{\mathcal{K}} \triangleq \max \left\{M,|\mathcal{K}| N_{t}\right\}$. We define $\lambda_{1} \leq \cdots \leq \lambda_{m_{\mathcal{K}}}$ to be the $m_{\mathcal{K}}$ ordered non-zero eigenvalues of $\mathbf{H}_{\mathcal{K}} \mathbf{H}_{\mathcal{K}}^{\mathrm{H}}$, and rewrite the above as

$$
R(\mathbf{H}) \leq \frac{1}{|\mathcal{K}|} \log \left[\prod_{i=1}^{m_{\mathcal{K}}}\left(1+\rho \lambda_{i}\right)\right]
$$

Shannon's source-channel separation theorem holds for a single-user system and any fixed channel. Therefore, as far as each of the above constraints are concerned, we have a situation completely equivalent to a single-user MIMO channel where the $|\mathcal{K}|$ users act as a single cooperative transmitter with a source of length $|\mathcal{K}| S$, operating over a MIMO channel with block length $T$ and channel coding rate $|\mathcal{K}| R(\mathbf{H})$. It follows that the corresponding distortion obtained by this genie-aided scheme is a lower bound on any achievable distortion for the actual MIMO-MAC. This is given by

$$
\begin{aligned}
D^{\mathrm{LB}}(\rho) & =\mathbb{E}[\exp (-b R(\mathbf{H}))] \\
& \geq \mathbb{E}\left[\prod_{i=1}^{m_{\mathcal{K}}}\left(1+\rho \lambda_{i}\right)^{-\frac{b}{|\mathcal{K}|}}\right] .
\end{aligned}
$$

We set ${ }^{3} \lambda_{i} \doteq \rho^{-\alpha_{i}}$. The joint pdf of the random vector $\boldsymbol{\alpha}=\left(\alpha_{1}, \ldots, \alpha_{m_{\mathcal{K}}}\right)$ is given as [1]

$$
p(\boldsymbol{\alpha}) \doteq\left\{\begin{array}{cc}
\rho^{-\sum_{i=1}^{m_{\mathcal{K}}}\left(2 i-1+n_{\mathcal{K}}-m_{\mathcal{K}}\right) \alpha_{i}}, & \alpha_{1} \geq \cdots \geq \alpha_{m_{\mathcal{K}}} \geq 0 \\
\rho^{-\infty}, & \text { otherwise }
\end{array}\right.
$$

Using (4) to compute (3) and an application of Varadhan's lemma as in [1] results in an upper-bound on the distortion SNR exponent

$$
\begin{aligned}
\delta^{*}(b) \leq \inf _{\alpha_{1} \geq \cdots \geq \alpha_{m_{\mathcal{K}} \geq 0}} \sum_{i=1}^{m_{\mathcal{K}}}\left[\frac{b}{|\mathcal{K}|}\left(1-\alpha_{i}\right)^{+}\right. \\
\left.+\left(2 i-1+n_{\mathcal{K}}-m_{\mathcal{K}}\right) \alpha_{i}\right],
\end{aligned}
$$

where $(x)^{+} \triangleq \max \{0, x\}$. It can be verified that the above infimum is achieved by

$$
\alpha_{i}^{*}=\left\{\begin{array}{ll}
0, & \frac{b}{|\mathcal{K}|} \leq 2 i-1+n_{\mathcal{K}}-m_{\mathcal{K}} \\
1, & \frac{b}{|\mathcal{K}|}>2 i-1+n_{\mathcal{K}}-m_{\mathcal{K}}
\end{array} .\right.
$$

${ }^{3}$ We use the exponential equality notation of [1],

$$
x \doteq y \Leftrightarrow \lim _{\rho \rightarrow \infty} \frac{\log x}{\log \rho}=\lim _{\rho \rightarrow \infty} \frac{\log y}{\log \rho}
$$


Hence

$$
\delta^{*}(b) \leq \sum_{i=1}^{m_{\mathcal{K}}} \min \left\{\frac{b}{|\mathcal{K}|}, 2 i-1+n_{\mathcal{K}}-m_{\mathcal{K}}\right\}
$$

$\forall \mathcal{K} \subseteq\{1, \ldots, K\}$. For a fixed $|\mathcal{K}|$, define

$$
\delta_{|\mathcal{K}|}(b)=\sum_{i=1}^{m_{\mathcal{K}}} \min \left\{\frac{b}{|\mathcal{K}|}, 2 i-1+n_{\mathcal{K}}-m_{\mathcal{K}}\right\} .
$$

We hence have that

$$
\delta^{*}(b) \leq \min \left\{\delta_{1}(b), \delta_{2}(b), \ldots, \delta_{K}(b)\right\}
$$

We term the above upper-bound as the informed transmitter bound.

We now investigate the informed transmitter bound for the important special case of $N_{t}=1$ and $M=K$. Notice that for $b \leq K$, we have that $\delta_{1}(b)=\delta_{K}(b)=b$. Let us examine how the $\delta_{|\mathcal{K}|}$ in (5) behave, for $1<|\mathcal{K}|<K$ in the range of $b \leq K$. In particular, we claim that

$$
\min \left\{\frac{b}{|\mathcal{K}|}, 2 i-1+K-|\mathcal{K}|\right\}=\frac{b}{|\mathcal{K}|}, i=1,2, \ldots,|\mathcal{K}| .
$$

This is true for $b \leq K$ since for some $\epsilon \geq 0$,

$$
\begin{array}{r}
2 i-1+K-|\mathcal{K}|<\frac{K}{|\mathcal{K}|}-\epsilon \\
\Leftrightarrow K<\frac{|\mathcal{K}|}{|\mathcal{K}|-1}(|\mathcal{K}|-2 i+1-\epsilon) \\
\Leftrightarrow K<|\mathcal{K}|-|\mathcal{K}| \frac{2 i-2+\epsilon}{|\mathcal{K}|-1},
\end{array}
$$

for $i=1,2, \ldots,|\mathcal{K}|$. Setting $i=1$, this requires that

$$
\begin{array}{r}
K<|\mathcal{K}|-\epsilon \frac{|\mathcal{K}|}{|\mathcal{K}|-1} \\
\Rightarrow K<|\mathcal{K}|
\end{array}
$$

which is not true. This implies that $\delta_{|\mathcal{K}|}(b)=b \forall b \leq K$ and $\forall 1 \leq|\mathcal{K}| \leq K$. In particular, $\delta_{|\mathcal{K}|}(K)=K$. Now consider the case $b>K$. Notice that $\delta_{1}(b)=K \forall b>K$. Since $\delta_{|\mathcal{K}|}(b)$ is an increasing function of $b$, we have that

$$
\begin{aligned}
\delta_{|\mathcal{K}|}(b) & \geq \delta_{|\mathcal{K}|}(K) \forall b \geq K \\
& =K
\end{aligned}
$$

This implies that

$$
\delta_{|\mathcal{K}|}(b) \geq \delta_{1}(b) \forall b .
$$

The informed transmitter bound hence reduces to

$$
\delta^{*}(b) \leq \delta_{1}(b)=\min \{b, K\} .
$$

\section{SeParated SOURCE-CHANNEL CODING FOR THE MIMO-MAC}

In this section, we compute the achievable distortion SNR exponent under separated source-channel coding. 


\section{A. Diversity multiplexing tradeoff (DMT) of the MIMO-MAC}

We first present a very brief overview of the DMT [1] of the MIMO-MAC [2], which is a metric of performance for channel coding. According to the DMT formulation, we scale the rate of transmission of the coding scheme $\mathcal{C}(\rho)$ as $r \log \rho$, where $r$ denotes the multiplexing gain. We define the diversity gain of the system to be

$$
d(r)=-\lim _{\rho \rightarrow \infty} \frac{\log P_{e}(\rho)}{\log \rho},
$$

where $P_{e}(\rho)$ denotes the codeword error probability. The DMT $d^{*}(r)$ is defined to be the supremum of all achievable diversity gains. We consider a common diversity requirement of $d(r)$ for the transmission of all UTs, and equal rate transmission. For the general $K$ user MAC with $N_{t}$ antennas at each UT and $M$ antennas at the BS, the DMT is given by [2]

$$
d_{M A C}^{*}(r)=\left\{\begin{array}{ll}
d_{N_{t}, M}^{*}(r), & r \leq \min \left\{N_{t}, \frac{M}{K+1}\right\} \\
d_{K N_{t}, M}^{*}(K r), & r \geq \min \left\{N_{t}, \frac{M}{K+1}\right\}
\end{array},\right.
$$

where $d_{n_{t}, n_{r}}^{*}(r)$ is the DMT for the single user MIMO channel with $n_{t}$ transmit and $n_{r}$ receive antennas [1], given by the piecewise linear function interpolating the points $\left(r,\left(n_{t}-r\right)\left(n_{r}-r\right)\right)$ for integral $r=0,1, \ldots, \min \left\{n_{t}, n_{r}\right\}$. The tradeoff performance can hence be divided into two regimes, the lightly loaded regime corresponding to $r \leq \min \left\{N_{t}, \frac{M}{K+1}\right\}$, and the heavily loaded regime corresponding to $r \geq \min \left\{N_{t}, \frac{M}{K+1}\right\}$. In the lightly loaded regime, the DMT of the MAC is as though there was only one user in the system, i.e., single-user performance is achieved. In the heavily loaded regime, the DMT of the MAC is as though all the users pooled their antennas together into a single "super-user", and transmit at $K$ times the single-user rate. The authors in [2] were also able to show that the dominant error event in the lightly loaded regime corresponds to a single user decoding in error, while the dominant error event in the heavily loaded regime is that of all users decoding in error.

\section{B. Distortion exponent with separated source-channel coding}

We now turn to the analysis of the distortion SNR exponent of a separated source-channel coding scheme for the MIMO-MAC. A separated source-channel coding scheme consists of concatenating a quantizer of rate $R_{s}$ bits/source sample with a channel code of rate $R_{c}$ bpcu, with $R_{s}=b R_{c}$. It was shown in [7] that the end-to-end distortion achievable by a separated scheme is upper-bounded by

$$
D_{\text {sep }}(\rho) \leq D_{Q}\left(R_{s}\right)+\kappa P_{e}(\rho)
$$

where $D_{Q}\left(R_{s}\right)$ denotes the quantizer distortion-rate function, $P_{e}(\rho)$ is the error probability of the channel code, and $\kappa$ is a constant independent of $\rho$. Let $R_{s}$ and $R_{c}$ denote the rates of the quantizer and the channel encoder respectively, with corresponding multiplexing gains $r_{s}$ and $r_{c}$. Thus,

$$
D_{s e p}(\rho) \leq \rho^{-b r_{c}}+\kappa \rho^{-d_{M A C}^{*}\left(r_{c}\right)} .
$$

Notice that there exist very simple scalar quantizers with rate-distortion optimal scaling $\rho^{-b r_{c}}[4]$, and DMT optimal codes with finite blocklength $K N_{t}+M-1$ [2]. The best possible distortion SNR exponent is obtained by choosing $r_{c}$ such that the two exponents of the above expression are balanced, i.e., such that $b r_{c}=d_{M A C}^{*}\left(r_{c}\right)$. These considerations lead to: 
Theorem 1: If $N_{t} \leq \frac{M}{K+1}$, or if $N_{t}>\frac{M}{K+1}$ and $b>\frac{K+1}{M} d_{N_{t}, M}^{*}\left(\frac{M}{K+1}\right)$, then the distortion SNR exponent of the separated source channel coding scheme

$$
\begin{array}{r}
\delta_{s e p}(b) \geq b \frac{j d_{N_{t}, M}^{*}(j-1)-(j-1) d_{N_{t}, M}^{*}(j)}{b+d_{N_{t}, M}^{*}(j-1)-d_{N_{t}, M}^{*}(j)}, \\
b \in\left(\frac{d_{N_{t}, M}^{*}(j)}{j}, \frac{d_{N_{t}, M}^{*}(j-1)}{j-1}\right],
\end{array}
$$

for $j=1, \ldots, \min \left\{N_{t}, M\right\}$. Else, the exponent is given by

$$
\delta_{\text {sep }}(b) \geq b \frac{j d_{K N_{t}, M}^{*}(j-1)-(j-1) d_{K N_{t}, M}^{*}(j)}{b-K^{2}\left[d_{K N_{t}, M}^{*}(j)-d_{K N_{t}, M}^{*}(j-1)\right]},
$$

for $b$ between $\frac{K d_{K N_{t}, M}^{*}(j)}{j}$ and $\frac{K d_{K N_{t}, M}^{*}(j-1)}{j-1}$, for $j=1, \ldots, \min \left\{M, \frac{N_{t}}{K}\right\}$.

Proof: For $N_{t} \leq \frac{M}{K+1}$, the MIMO-MAC DMT coincides with the single-user DMT for all $r$. The optimal distortion exponent in this case is the same as that for the single-user case, obtained in [4]. For the case when $N_{t}>\frac{M}{K+1}$, the transition between the lightly loaded and the heavily loaded regimes occurs at $r_{c}=\frac{M}{K+1}$, at which point the diversity gain can be shown to be

$$
\begin{aligned}
d_{N_{t}, M}^{*}\left(\frac{M}{K+1}\right)= & \left(\frac{M}{K+1}-\left\lfloor\frac{M}{K+1}\right\rfloor\right)\left[2\left\lfloor\frac{M}{K+1}\right\rfloor-\left(N_{t}+M-1\right)\right] \\
& +\left(N_{t}-\left\lfloor\frac{M}{K+1}\right\rfloor\right)\left(M-\left\lfloor\frac{M}{K+1}\right\rfloor\right) .
\end{aligned}
$$

The slope of the line connecting the origin with $\left(\frac{M}{K+1}, d_{N_{t}, M}^{*}\left(\frac{M}{K+1}\right)\right)$ is $\frac{K+1}{M} d_{N_{t}, M}^{*}\left(\frac{M}{K+1}\right)$. Hence if $b>\frac{K+1}{M} d_{N_{t}, M}^{*}\left(\frac{M}{K+1}\right)$, then once again the single-user solution from [4] holds. For the case when $b<\frac{K+1}{M} d_{N_{t}, M}^{*}\left(\frac{M}{K+1}\right)$, we need to solve

$$
b r_{c}=d_{K N_{t}, M}^{*}\left(K r_{c}\right) .
$$

For $r_{c}=\frac{j}{K}, j=0,1, \ldots, \min \left\{M, \frac{N_{t}}{K}\right\}$, we have

$$
b=\frac{K d_{K N_{t}, M}^{*}(j)}{j}
$$

Then $\delta_{\text {sep }}(b)$ interpolates the points $\left(\frac{K d_{K N_{t}, M}^{*}(j)}{j}, d_{K N_{t}, M}^{*}(j)\right)$ for $j=0,1, \ldots, \min \left\{M, \frac{N_{t}}{K}\right\}$.

For $r_{c} \in\left[\frac{j-1}{K}, \frac{j}{K}\right]$, the function $d_{K N_{t}, M}^{*}\left(K r_{c}\right)$ linearly interpolates the points $\left(\frac{j-1}{K}, d_{K N_{t}, M}^{*}(j-1)\right)$ and $\left(\frac{j}{K}, d_{K N_{t}, M}^{*}(j)\right)$. This gives us that

$$
\begin{aligned}
d_{K N_{t}, M}^{*}\left(K r_{c}\right)= & K\left(d_{K N_{t}, M}^{*}(j)-d_{K N_{t}, M}^{*}(j-1)\right)\left[K r_{c}-\frac{j-1}{K}\right]+d_{K N_{t}, M}^{*}(j-1), \\
& \text { for } r_{c} \in\left[\frac{j-1}{K}, \frac{j}{K}\right]
\end{aligned}
$$

Solving (6), we hence obtain the exponent

$$
b r_{c}=b \frac{j d_{K N_{t}, M}^{*}(j-1)-(j-1) d_{K N_{t}, M}^{*}(j)}{b-K^{2}\left[d_{K N_{t}, M}^{*}(j)-d_{K N_{t}, M}^{*}(j-1)\right]},
$$

for $b$ between $K \frac{d_{K N_{t}, M}^{*}(j)}{j}$ and $K \frac{d_{K N_{t}, M}^{*}(j-1)}{j-1}$.

For the special case when $N_{t}=1$ and $M=K, d_{M A C}^{*}\left(r_{c}\right)=K\left(1-r_{c}\right)$, leading us to choose

$$
r_{c}=\frac{K}{K+b} \Rightarrow \delta_{s e p}(b) \geq \frac{b K}{K+b} \text {. }
$$




\section{Performance of successive interference cancellation (SIC) receivers}

We now consider the performance of low complexity MMSE and ZF SIC receivers (i.e., MMSE and ZF V-BLAST receivers [10]) in terms of distortion SNR exponents. We show that these low complexity receivers are very suboptimal (irrespective of the order in which users are decoded), which suggests that joint decoding techniques might be inevitable in this scenario. The suboptimality in terms of distortion exponent is a direct consequence of the suboptimality of both MMSE and ZF SIC receivers in terms of DMT. For simplicity of exposition we restrict ourself to the case of $N_{t}=1$ and $M=K$. It was shown in [2] that these receivers with no user ordering achieve a DMT of $d_{S I C}^{*}(r)=1-r_{c}$. It was later shown in [11] that no ordering, including the V-BLAST optimal ordering [10] can improve upon this DMT. In the separated case, this would result in a very suboptimal distortion-SNR exponent of

$$
\delta_{\text {sep }, S I C}(b)=\frac{b}{1+b} .
$$

Since $\delta(b) \geq 1$ is needed in order to achieve bounded or vanishing rate gap for the MIMO downlink (see Sec. III), we conclude that a digital "separated" scheme based on SIC fails to achieve this goal, and would result inevitably in a loss of degrees of freedom in the MIMO downlink due to the poor SNR exponent of the CSIT feedback.

\section{Hybrid Digital-Analog Coding Scheme for the MiMO-MAC}

While the separated scheme is close to optimal for very low and very high bandwidth efficiencies, it is in fact very suboptimal for a wide range of bandwidth efficiencies, including the ones that are most relevant for practical CSIT feedback scheme, such as $b$ between 2 and 6 . One therefore needs to consider joint source channel coding to improve upon the performance of the separated source channel coding scheme. This approach has been employed with good success in the case of the single-user MIMO channel in the series of works in [4]-[6]. In the following subsection, we consider a generalization of the hybrid digital-analog scheme of [4] for the MIMO-MAC, and evaluate the distortion SNR exponent for this case.

We restrict our attention to the case of bandwidth expansion, i.e., $b \geq 1$ (the case of bandwidth compression $b<1$ is not relevant for our purpose since it would result in a loss of downlink multiplexing gain [9]). Along the lines of [4], we consider the encoder shown in Fig. 1 for each UT.

We assume that $M \geq K$ in this section. Define $N_{t}^{\prime}$ to be the maximum integer less than or equal to $N_{t}$ such that $K N_{t}^{\prime} \leq M$. The block of $S$ input symbols corresponding to the $i^{\text {th }} \mathrm{UT}$ is first fed to a separated source-channel encoder that produces "digital" encoded data $\mathbf{X}_{i}^{d}$ of size $N_{t} \times T_{d}$. The quantization error e is scaled by $1 / \sqrt{N_{t}^{\prime} D_{Q}\left(R_{s}\right)}$ and reformatted to produce "analog" data $\mathbf{X}_{i}^{a}$ of size $N_{t}^{\prime} \times S / N_{t}^{\prime}$. The analog data is to be transmitted over a pre-selected set of $N_{t}^{\prime}$ antennas. The digital and analog data are multiplexed in time to produce the signal $\mathbf{X}_{i}$ transmitted by the $i^{\text {th }} \mathrm{UT}$, over $T=T_{d}+S / N_{t}^{\prime}$ time-slots. We have that

$$
r_{c}=\frac{S}{T_{d}} r_{s} \quad \text { and } \quad b=\frac{T_{d}+S / N_{t}^{\prime}}{S},
$$

which result in

$$
r_{c}=\frac{N_{t}^{\prime} r_{s}}{N_{t}^{\prime} b-1}
$$

The BS receives a faded superposition of the signals transmitted by the various UT in AWGN, according to (1). The BS first tries to decode the digital data, by using the first $T_{d}$ columns of $\mathbf{Y}$, denoted as $\mathbf{Y}^{d}$. Up 


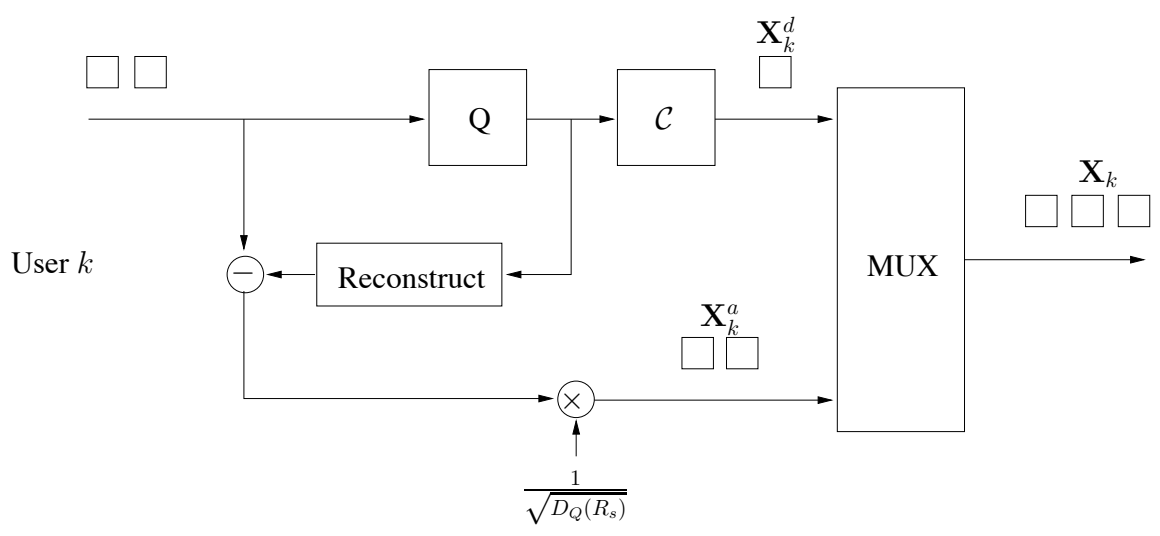

Fig. 1. Hybrid digital-analog scheme for bandwidth expansion

to SNR exponents, the probability of error can be replaced with the probability of outage for the MAC. The outage event is given by $\mathcal{O}=\left\{\left\{\mathbf{H}_{i}\right\}_{i=1}^{K}:\left(R_{c}, \ldots, R_{c}\right) \notin \mathcal{C}_{\mathrm{MAC}}(\mathbf{H} ; \rho)\right\}$.

If the decoding is successful, the BS obtains a linear MMSE estimate of the estimation error e from the last $S / N_{t}^{\prime}$ columns of $\mathbf{Y}$ (denoted as $\mathbf{Y}^{a}$ ), and adds it to the decoded digital information to produce a reconstruction. The following theorem presents the distortion SNR exponent for this hybrid digital-analog coding scheme (while an explicit solution may be obtained along the lines of Theorem 1, we refrain from the details in the general case for reasons of brevity, and present the closed-form solution only for the case of $\left.N_{t}=1, M=K\right)$.

Theorem 2: The distortion SNR exponent for the hybrid digital-analog coding scheme for the MAC is given by

$$
\delta_{h y b}(b) \geq d_{M A C}^{*}\left(r^{*}\right)
$$

where $r^{*}$ is the value of $r_{c}$ that solves

$$
1+\frac{r_{c}}{N_{t}^{\prime}}\left(N_{t}^{\prime} b-1\right)=d_{M A C}^{*}\left(r_{c}\right)
$$

and $N_{t}^{\prime}$ is the maximum integer less than or equal to $N_{t}$ such that $K N_{t}^{\prime} \leq M$. In particular, when $N_{t}=1$ and $M=K$ the distortion SNR exponent is given by

$$
\delta_{\text {hybrid }}(b) \geq 1+\frac{(b-1)(K-1)}{K+b-1} .
$$

Proof: Let $\mathbf{H}_{k}^{a}$ denote the $M \times N_{t}^{\prime}$ channel from the $k^{\text {th }}$ UT to the BS corresponding to the $N_{t}^{\prime}$ antennas chosen for analog transmission and $\mathbf{H}^{a} \triangleq\left[\begin{array}{lll}\mathbf{H}_{1}^{a} & \cdots & \mathbf{H}_{K}^{a}\end{array}\right]$. Notice that

$$
\begin{aligned}
\mathbf{Y}^{a} & =\sqrt{\rho} \sum_{k=1}^{K} \mathbf{H}_{k}^{a} \mathbf{X}_{k}+\mathbf{W}^{a} \\
& =\sqrt{\rho} \underbrace{\left[\mathbf{H}_{1}^{a} \mathbf{H}_{2}^{a} \cdots \mathbf{H}_{K}^{a}\right]}_{\triangleq \mathbf{H}^{a}} \underbrace{\left[\begin{array}{c}
\mathbf{X}_{1}^{a} \\
\mathbf{X}_{2}^{a} \\
\vdots \\
\mathbf{X}_{K}^{a}
\end{array}\right]}_{\triangleq \mathbf{X}^{a}}+\mathbf{W}^{a} .
\end{aligned}
$$


Stacking the columns of $\mathbf{Y}^{a}$ and $\mathbf{X}^{a}$ one below the other to obtain $\mathbf{y}^{a}$ and $\mathbf{x}^{a}$ respectively, we obtain the equivalent vector model

$$
\mathbf{y}^{a}=\sqrt{\rho} \underbrace{\left[\begin{array}{cccc}
\mathbf{H}^{a} & & & \\
& \mathbf{H}^{a} & & \\
& & \ddots & \\
& & \mathbf{H}^{a}
\end{array}\right]}_{\triangleq \mathbf{H}_{e q}=\mathbf{I}_{S / N_{t}^{\prime}} \otimes \mathbf{H}^{a}} \mathbf{x}^{a}+\mathbf{w}^{a}
$$

Notice that we may obtain an LMMSE estimate of $\mathbf{e}$ by first linearly estimating $\mathbf{x}^{a}$ from $\mathbf{y}^{a}$, and then by rearranging components and scaling. In order to estimate $\mathbf{x}^{a}$ from $\mathbf{y}^{a}$, we compute the covariance matrices

$$
\begin{aligned}
K_{\mathbf{x}^{a} \mathbf{x}^{a *}} & =\mathbf{I} \\
K_{\mathbf{x}^{a} \mathbf{y}^{a *}} & =\sqrt{\rho} \mathbf{H}_{e q}^{\mathrm{H}} \\
K_{\mathbf{y}^{a} \mathbf{y}^{a *}} & =\mathbf{I}+\rho \mathbf{H}_{e q} \mathbf{H}_{e q}^{\mathrm{H}} .
\end{aligned}
$$

From the orthogonality principle, the minimum mean squared error

$$
\begin{aligned}
\operatorname{mmse}\left(\mathbf{x}^{a} \mid \mathbf{H}\right) & \doteq \operatorname{tr}\left(\mathbf{I}-\rho \mathbf{H}_{e q}^{\mathrm{H}}\left(\mathbf{I}+\rho \mathbf{H}_{e q} \mathbf{H}_{e q}^{\mathrm{H}}\right)^{-1} \mathbf{H}_{e q}\right) \\
& =\operatorname{tr}\left[\left(\mathbf{I}+\rho \mathbf{H}_{e q}^{\mathrm{H}} \mathbf{H}_{e q}\right)^{-1}\right] \\
& =\sum_{k=1}^{m^{\prime}} \frac{S / N_{t}^{\prime}}{1+\rho \lambda_{k}}
\end{aligned}
$$

where $m^{\prime} \triangleq \min \left\{M, K N_{t}^{\prime}\right\}$ and $\lambda_{1} \leq \cdots \leq \lambda_{m^{\prime}}$ denote the ordered eigenvalues of $\mathbf{H}^{\mathrm{H}} \mathbf{H}$. Thus

$$
\operatorname{mmse}(\mathbf{e} \mid \mathbf{H})=D_{Q}\left(R_{s}\right) \sum_{k=1}^{m^{\prime}} \frac{S / N_{t}^{\prime}}{1+\rho \lambda_{k}} .
$$

It can be shown that the distortion for the hybrid scheme is given by

$$
\begin{aligned}
D_{\text {hybrid }}(\rho) & \leq \int_{\mathcal{O}^{c}} \operatorname{mmse}(\mathbf{e} \mid \mathbf{H}) P(\mathbf{H}) d \mathbf{H}+\kappa P(\mathcal{O}) \\
& \leq \int \operatorname{mmse}(\mathbf{e} \mid \mathbf{H}) P(\mathbf{H}) d \mathbf{H}+\kappa P(\mathcal{O})
\end{aligned}
$$

where $\kappa$ is a constant independent of $\rho$.

Let $\lambda_{i} \doteq \rho^{-\alpha_{i}}$. Using the joint pdf $p(\boldsymbol{\alpha})$ given in (4), we compute

$$
\begin{aligned}
\int \mathrm{mmse}(\mathbf{e} \mid \mathbf{H}) P(\mathbf{H}) d \mathbf{H} & \doteq \rho^{-r_{s}} \int_{\alpha_{i} \geq 0} \rho^{-\left(1-\alpha_{1}\right)^{+}} \rho^{-\sum_{i=1}^{m^{\prime}}\left(2 i-1+\left|M-K N_{t}^{\prime}\right|\right) \alpha_{i}} d \boldsymbol{\alpha} \\
& \doteq \rho^{-r_{s}} \rho^{-\inf _{i} \geq 0 \forall i} f(\boldsymbol{\alpha})
\end{aligned}
$$

where $f(\boldsymbol{\alpha})=\left(1-\alpha_{1}\right)^{+}+\sum_{i=1}^{m^{\prime}}\left(2 i-1+\left|M-K N_{t}^{\prime}\right|\right) \alpha_{i}$. Hence

$$
\int \operatorname{mmse}(\mathbf{e} \mid \mathbf{H}) P(\mathbf{H}) d \mathbf{H} \doteq \rho^{-\left(1+r_{s}\right)} .
$$

The distortion SNR exponent for the hybrid scheme is obtained by equating the SNR exponents of the two terms in $(8)$. 


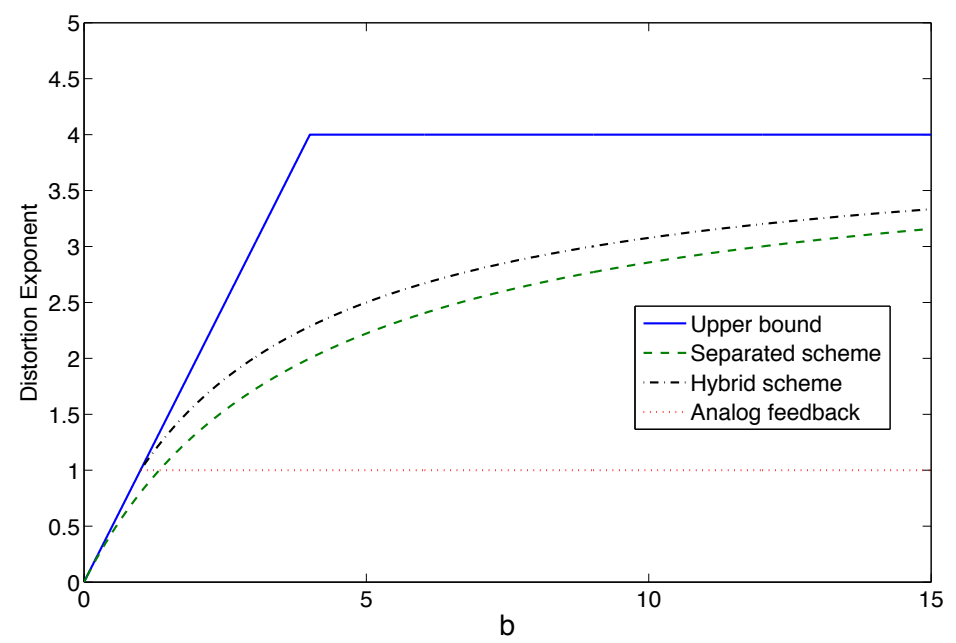

Fig. 2. Distortion SNR exponent for the MIMO-MAC, $N_{t}=1, M=K$

Similar to the separated source channel coding case, it can be shown that successive interference cancellation is very suboptimal in this case also. Fig. 2 shows plots of the distortion exponent for $K=4$ obtained from separated source channel coding and hybrid digital-analog coding in comparison to the informed transmitter upper bound and the analog scheme.

\section{Optimal Channel Code Design for Single Antenna User Terminals}

In order to achieve the distortion SNR exponents computed in Theorems 1 and 2, a DMT optimal code for the MIMO MAC is needed. For applications such as CSIT feedback over cellular networks, it is very important to design such codes that are simple to both encode and decode. Further, designing codes with low latency is of paramount importance, both to reduce the feedback overhead, and to ensure that the estimated channel values at the base station are not outdated. Hence strategies such as employing high-dimensional quantizers that create large blocks of quantized bits and then mapping them into channel codes with large block length (e.g., LDPC codes) are ruled out for this application. Keeping this in mind, for the practically important case of $N_{t}=1$ and $M=K$, we propose to use simple scalar quantization and uncoded QAM modulation. While simulation results in the subsequent section will show that this is indeed a very good strategy in terms of performance, the objective of this section will be to show that uncoded QAM is indeed DMT optimal for the MIMO-MAC with single antenna UTs.

Theorem 3: Consider a MAC with $N_{t}=1$ and $M=K$ where the users transmit uncoded QAM symbols (i.e., $T=1$ ) at a per-user multiplexing gain of $r$, and the receiver employs a joint maximum likelihood decoder. This scheme achieves the optimal DMT of $M(1-r)$ for the MAC.

In order to prove this theorem, we will make use of the following lemma from [12].

Lemma 4: Let $\mathrm{G}$ be an $N \times M$ matrix whose entries are generated i.i.d. $\mathcal{C N}(0,1)$. Denote the minimum distance of the lattice generated by $\mathbf{G}$ to be $d_{\mathbf{G}}$. Then

$$
P\left\{d_{\mathbf{G}} \leq \epsilon\right\} \leq\left\{\begin{array}{cc}
\beta_{N, M} \epsilon^{2 N}, & M<N \\
\beta_{N, N} \epsilon^{2 N} \max \left\{(-\ln \epsilon)^{N+1}, 1\right\}, & M=N
\end{array} .\right.
$$


We now prove Theorem 3 .

Proof: (Theorem 3) Consider the following linear Gaussian channel model that is equivalent to (1),

$$
\mathbf{y}=\theta \mathbf{H x}+\mathbf{w}
$$

where $\mathbf{H} \in \mathbb{C}^{M \times M}$ and $\mathbf{x}, \mathbf{y}, \mathbf{w} \in \mathbb{C}^{M \times 1}$. Let the entries of $\mathbf{H}, \mathbf{w}$ be drawn i.i.d. from a $\mathcal{C N}(0,1)$ distribution. The codeword $\mathbf{x}$ corresponds to uncoded QAM, where each component is drawn from the following $Q^{2}$-QAM alphabet:

$$
\{a+\imath b \mid a, b \in\{-Q+1, \ldots,-1,1, \ldots, Q-1\}\} .
$$

Further, we choose $\theta$ such that $\mathbb{E}\left\{|\theta \mathbf{x}|^{2}\right\} \doteq \rho$. We assume a per stream rate of $R=r \log \rho$, i.e., we have $Q^{2} \doteq \rho^{r}$. Since $\mathbb{E}\left\{|\theta \mathbf{x}|^{2}\right\} \doteq Q^{2} \theta^{2}$, this gives us that $\theta^{2} \doteq \rho^{1-r}$.

Let $d_{\text {min }}$ denote the minimum distance of the lattice corresponding to $\theta \mathbf{H x}$. We may write the error probability involved in jointly decoding $\mathbf{x}$ from $\mathbf{y}$ as

$$
P_{e}=P\left(\operatorname{error} \mid d_{\min } \dot{\leq} \rho^{0}\right) P\left(d_{\min } \dot{\leq} \rho^{0}\right)+P\left(\operatorname{error} \mid d_{\min } \dot{>} \rho^{0}\right) P\left(d_{\min } \dot{>} \rho^{0}\right) .
$$

Now consider the term $P\left(\right.$ error $\left.\mid d_{\text {min }}>\rho^{0}\right)$. Given that the minimum distance $d_{\text {min }}$ of the received signal lattice $\theta \mathbf{H x}$ is exponentially greater than $\rho^{0}$, the minimum distance ML decoder will always decode to the correct transmitted codeword if the magnitude of the noise is exponentially smaller than $\rho^{0}$. This gives us that

$$
P\left(\operatorname{error} \mid d_{\min } \dot{>} \rho^{0}\right) \leq P\left(|\mathbf{w}| \geq \frac{\rho^{\epsilon}}{2}\right),
$$

for some $\epsilon>0$. Since $|\mathbf{w}|^{2}$ is a chi-squared random variable in $2 M$ dimensions, we can simplify the above using the expression for the cdf of a chi-squared distribution [3] as

$$
\begin{aligned}
P\left(\operatorname{error} \mid d_{\min }>\rho^{0}\right) & \leq \exp \left(-\frac{\rho^{2 \epsilon}}{4}\right) \sum_{k=0}^{M} \frac{\left(\frac{\rho^{2 \epsilon}}{4}\right)^{k}}{k !} \\
& \doteq \exp \left(-\rho^{2 \epsilon}\right)
\end{aligned}
$$

for some $\epsilon>0$. Using this in (10), we obtain that

$$
\begin{aligned}
P_{e} & \doteq P\left(\text { error } \mid d_{\min } \dot{\leq} \rho^{0}\right) P\left(d_{\min } \dot{\leq} \rho^{0}\right) \\
& \leq P\left(d_{\min } \dot{\leq} \rho^{0}\right)
\end{aligned}
$$

Noticing that $d_{\min }=\theta d_{\mathbf{H}}$, we obtain from (11) and an application of Lemma 4 that

$$
\begin{aligned}
P_{e} & \leq P\left(d_{\mathbf{H}} \leq \frac{1}{\rho^{(1-r) / 2}}\right) \\
& \doteq \frac{1}{\rho^{M(1-r)}}(\ln \rho)^{n+1} \\
& \doteq \rho^{-M(1-r)}
\end{aligned}
$$

Remark 1: A closer look at the proof of Theorem 3 will reveal that the same proof will carry over unchanged to the case where we use a minimum distance lattice decoder. By a minimum distance lattice 


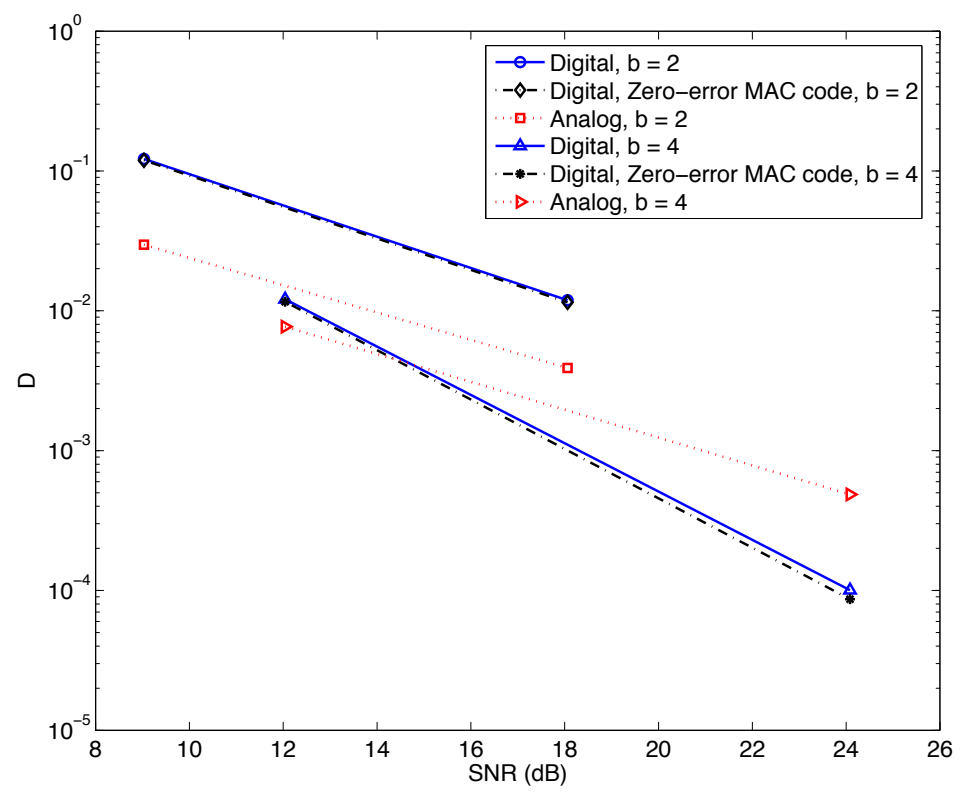

Fig. 3. Comparing analog feedback with digital feedback using separated source-channel coding, $K=M=4, N_{t}=1$

decoder, we mean a sphere decoder [14] that decodes to the whole infinite lattice and not just the finite constellation carved from the lattice, without doing any special precoding or weighting. Such a decoder is referred to as a "naive lattice decoder" in [13], in contrast to the MMSE-GDFE lattice decoder [13], or the regularized lattice decoder [15].

\section{SimULATION RESUlTS}

In this section, we present simulation results comparing the case of digital and analog CSIT feedback over the MIMO-MAC. We focus on the case of $N_{t}=1$ and $K=M=4$ and restrict attention to separated source channel coding for the digital feedback case. Further, we focus on the following extremely simple encoder, comprising of a uniform scalar quantizer [4] followed by uncoded QAM for the channel code (these are respectively optimal with respect to quantizer distortion, and DMT). We will restrict attention to the case of joint ML decoding at the receiver (implemented using a sphere decoder [14]).

Fig. 3 compares the per-user squared error distortion using analog and digital feedback for the cases of $b=2$ and $b=4$. For the analog feedback case, we use time-division with 2 users transmitting un-quantized Gaussian sources simultaneously while satisfying the average power constraint (such a timedivision strategy has been proven to be the optimal strategy for analog feedback [9]). Notice that we fix $T=4$ for the digital case, and vary the number of samples of the transmitted Gaussian source $S=2,1$ to achieve $b=2,4$ respectively. We vary the codebook size with SNR according to the optimal multiplexing gains $r_{c}=2 / 3$ and $r_{c}=1 / 2$ corresponding to $b=2,4$ respectively, computed using (7). For example, at $b=4$, this would correspond to 4-QAM and 16-QAM constellations at SNRs of $12.0412 \mathrm{~dB}$ and 24.0824 $\mathrm{dB}$ respectively (in turn corresponding to rates $R_{c}=2,4 \mathrm{bpcu}$ and $R_{s}=8,16$ bits/complex sample respectively). We make use of scalar uniform quantizers from [4] and use gray maps to assign bits to the QAM alphabets. While the slope of analog feedback can never exceed 1, we see that the slopes of the digital feedback in Fig 3 are close to their theoretical values of $\delta_{\text {sep }}(2) \geq \frac{4}{3}$ and $\delta_{\text {sep }}(4) \geq 2$. While analog feedback is better at $b=2$, we observe that the digital feedback significantly outperforms analog 


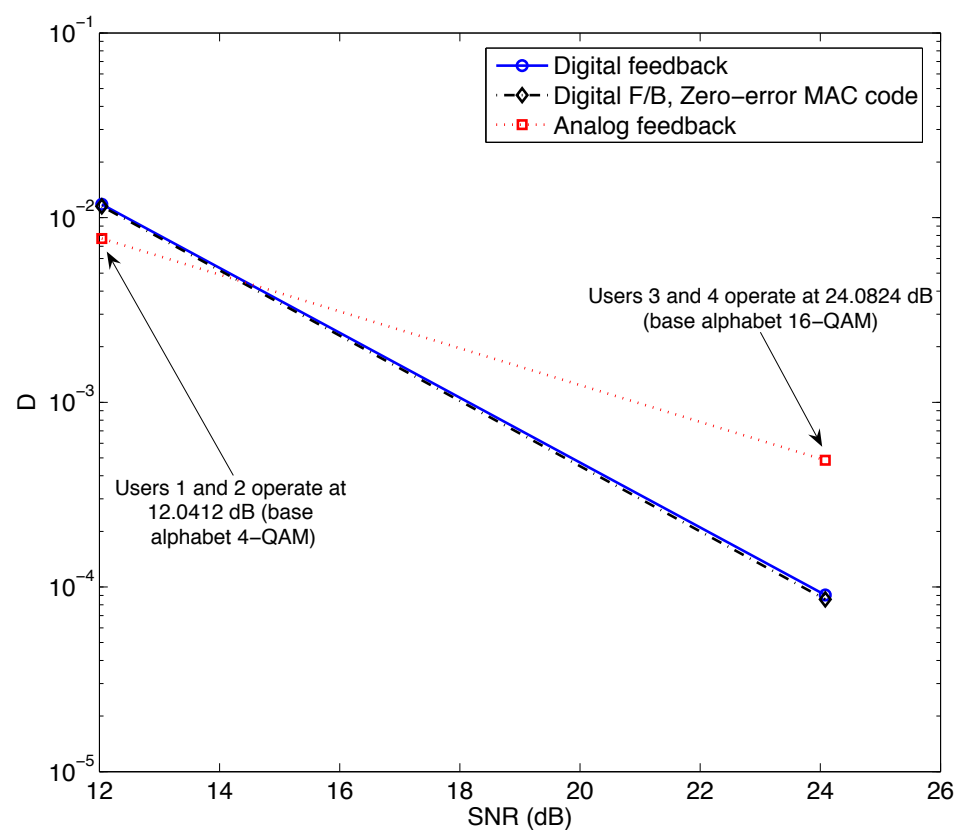

Fig. 4. Distortion obtained for users with very different SNRs, $K=M=4, N_{t}=1$

feedback at $b=4$. In order to illustrate that the channel code in this example is indeed near optimal, we also plot the distortion achieved by an ideal zero-error MAC code, for which the only distortion is due to the source quantization. We observe that the distortion performance of the chosen MAC code is almost identical to the ideal zero-error case. This does not mean that uncoded QAM achieves zero error at these values of SNRs, of course. It just indicates that the probability of error scales with SNR together with the quantization distortion, such that the performance is effectively given by the quantization distortion, in agreement with the optimization of the SNR exponent in Sections IV and V]

In a practical cellular system, the SNR experienced by UTs close to the BS and those at the cell fringes may differ by several tens of $\mathrm{dBs}$, owing to significantly different path-loss. As we saw before, the downlink rate-gap is determined by $\rho D(\rho)$, which necessitates that the distortion decays with an exponent strictly larger than 1 to ensure a vanishing rate gap. Analog feedback is insufficient for this purpose, leading to a rate-gap that does not decay with SNR (the presence of sub-logarithmic terms results in the exponent being slightly less than one in practice). Digital feedback is hence a necessity in this practical scenario. Corresponding to this scenario, we present simulations of a $K=4$ user system, where two high-SNR users operate at $24 \mathrm{~dB}$, and two low-SNR users operate at $12 \mathrm{~dB}$. We fix $b=4$, and choose the rates of the users in accordance with the distortion-optimal multiplexing gain of $r_{c}=1 / 2$ (hence low SNR users signal using 4-QAM and high SNR users with 16-QAM). We plot the per-user distortions obtained for the low and high SNR users against their respective SNRs in Fig. 4, and observe that the distortion decays with a slope that is exactly equal to the slope for the case of $b=4$ in Fig. 3. This provides a very strong motivation for the use of intelligently designed digital CSIT feedback schemes in practice, and is also testament to the practical relevance of the theoretical distortion exponents derived in this paper. 


\section{CONCLUSIONS}

In this paper, we considered the very important practical problem of designing CSIT feedback schemes for cellular systems. We modeled this problem as the transmission of Gaussian sources over a MIMOMAC, and argued that an appropriate metric to consider in order to maximize the downlink rate in the cellular system is the mean squared error distortion at which the channel is estimated at the base station. An upper bound was obtained on the asymptotic SNR-exponent of the distortion, and some CSIT feedback strategies were compared in terms of their achievable distortion SNR exponent. While traditionally, system designers have considered using analog (unquantized) feedback, we showed that such a strategy can at best lead only to an achievable downlink rate that is at a constant gap from the downlink rate achievable with perfect CSIT. We designed two "digital" feedback schemes, using separated and joint source channel coding respectively, that lead to an achievable downlink rate that approaches the optimal CSI downlink rate as the SNR increases (i.e., the rate gap vanishes).

We then turned our attention to another very important system design problem: the design of explicit quantization and channel coding schemes for the proposed digital feedback scheme. Simplicity of such schemes is very important for the application of CSIT feedback: complicated structures employing long outer codes and high-dimensional vector quantizers would increase the feedback overhead considerably, and the resulting high latency (large blocklengths for the feedback scheme) would render the CSIT fed back completely outdated and hence useless for the purpose of multiuser MIMO downlink precoding. We showed that a very simple separated scheme comprising of a uniform scalar quantizer and uncoded QAM modulation is optimal in terms of distortion SNR exponent for the case of single antenna user terminals. Further, we demonstrated through simulations that such a scheme outperforms analog feedback in practical scenarios. Our simulations also reveal the advantage of using variable rate feedback for users with different operating SNRs in a cellular system with large pathloss dynamic range. A naive feedback scheme with a fixed number of bits would result in an interference-limited system, while our digital feedback scheme results in significant performance gains, especially for the users in very good SNR conditions.

\section{REFERENCES}

[1] L. Zheng and D. Tse, "Diversity and Multiplexing: A Fundamental Tradeoff in Multiple-Antenna Channels," IEEE Trans. Inform. Theory, Vol. 49, No. 5, pp. 1073-1096, May 2003.

[2] D. Tse, P. Viswanath and L. Zheng , "Diversity-multiplexing tradeoff in multiple-access channels," IEEE Trans. Inform. Theory, Vol. 50, No. 9, pp. 1859 - 1874, Sep. 2004.

[3] J. G. Proakis, Digital Communications, McGraw-Hill International, New York, 2001.

[4] G. Caire and K. R. Narayanan, "On the Distortion SNR Exponent of Hybrid DigitalAnalog SpaceTime Coding," IEEE Trans. Inform. Theory, vol. 53, no. 8, pp. 2867 - 2878, Aug. 2007.

[5] K. Bhattad, K. R. Narayanan and G. Caire, "On the Distortion SNR Exponent of Some Layered Transmission Schemes," IEEE Trans. Inform. Theory, vol. 54, no. 7, pp. 2943 - 2958, Jul. 2008.

[6] D. Gunduz and E. Erkip, “Joint Source-Channel Codes for MIMO Block-Fading Channels," IEEE Trans. Inform. Theory, vol. 54, no. 1, Jan. 2008.

[7] B. Hochwald and K. Zeger, "Tradeoff between source and channel coding," IEEE Trans. Inform. Theory, vol. 43, no. 5, May 1997.

[8] N. Jindal, "MIMO broadcast channels with finite rate feedback," IEEE Trans. Inform. Theory, vol. 52, no. 11, pp. 5045 - 5059, Nov. 2006.

[9] G. Caire, N. Jindal, M. Kobayashi and N. Ravindran, "Multiuser MIMO Downlink Made Practical: Achievable Rates with Simple Channel State Estimation and Feedback Schemes," Submitted to IEEE Trans. Inform. Theory, Arxiv preprint arXiv:0711.2642, Nov. 2007.

[10] P. W. Wolniansky, G. J. Foschini, G. D. Golden and R. A. Valenzuela, "V-BLAST: An Architecture for Realizing Very High Data Rates Over the Rich-Scattering Wireless Channel,” invited paper, Proc. ISSSE-98, Pisa, Italy, Sept. 29, 1998. 
[11] Y. Jiang, M. Varanasi, and J. Li, "Performance analysis of ZF and MMSE equalizers for MIMO systems: A closer study in high SNR regime," IEEE Trans. Inform. Theory, submitted Aug. 2006, and accepted for publication.

[12] M. Taherzadeh, A. Mobasher and A. K. Khandani, "LLL Reduction Achieves the Receive Diversity in MIMO Decoding," IEEE Trans. Inform. Theory, Vol. 53, No. 12, pp. 4801-4805, Dec. 2007.

[13] H. El Gamal, G. Caire and M.O. Damen, "Lattice Coding and Decoding Achieve the Optimal Diversity-Multilpexing Tradeoff of MIMO Channels," IEEE Trans. Inform. Theory, Vol. 50, No. 6, pp. 968-985, June 2004.

[14] M. O. Damen, H. El Gamal and G. Caire, "On maximum likelihood detection and the search for the closest lattice point," IEEE Trans. Inform. Theory, Vol. 49, No. 10, pp. 2389-2402, Oct. 2003.

[15] Joakim Jalden and Petros Elia, "DMT Optimality of LR-Aided Linear Decoders for a General Class of Channels, Lattice Designs, and System Models," Submitted to IEEE Trans. Inform. Theory, May 2009. arXiv:0905.4023v1 [cs.IT]. 